\title{
Synthesis of indium nanoparticles at ambient temperature; simultaneous phase transfer and ripening
}

\author{
Mohammad Aghazadeh Meshgi • Manfred Kriechbaum • Subhajit Biswas • \\ Justin D. Holmes • Christoph Marschner $\mathbb{D}$
}

Received: 23 June 2016 / Accepted: 21 November 2016/Published online: 5 December 2016

(C) The Author(s) 2016. This article is published with open access at Springerlink.com

\begin{abstract}
The synthesis of size-monodispersed indium nanoparticles via an innovative simultaneous phase transfer and ripening method is reported. The formation of nanoparticles occurs in a one-step process instead of well-known two-step phase transfer approaches. The synthesis involves the reduction of $\mathrm{InCl}_{3}$ with $\mathrm{LiBH}_{4}$ at ambient temperature and although the reduction occurs at room temperature, fine indium nanoparticles, with a mean diameter of $6.4 \pm 0.4 \mathrm{~nm}$, were obtained directly in non-polar $n$-dodecane. The direct synthesis of indium nanoparticles in $n$-dodecane facilitates their fast formation and enhances their size-monodispersity. In addition, the nanoparticles were highly stable for more than 2 months. The nanoparticles were characterised by dynamic light scattering (DLS), small angle X-ray scattering (SAXS), transmission electron microscopy (TEM), energy dispersive X-ray spectroscopy (EDS) and Fourier transform infrared (FT-IR) spectroscopy to determine their morphology, structure and phase purity.
\end{abstract}

\footnotetext{
M. Aghazadeh Meshgi • M. Kriechbaum •

C. Marschner $(\bowtie)$

Institute of Inorganic Chemistry, Graz University of Technology, Stremayrgasse 9, Graz, Austria

e-mail: christoph.marschner@tugraz.at

S. Biswas · J. D. Holmes $(\bowtie)$

Materials Chemistry and Analysis Group, Department of

Chemistry and the Tyndall National Institute, University College

Cork, Cork, Ireland

e-mail: j.holmes@ucc.ie

S. Biswas · J. D. Holmes

AMBER@CRANN, Trinity College Dublin, Dublin 2, Ireland
}

Keywords Indium $\cdot$ Nanoparticle $\cdot$ Monodisperse . Colloidal solution $\cdot$ Reduction synthesis $\cdot$ SAXS

\section{Introduction}

Indium nanoparticles have been widely used as seeding particles for the growth of III-V semiconductor nanowires via solution or vapour-liquid-solid (VLS) mechanisms (Wang et al. 2006; Strupeit et al. 2009; Zhang et al. 2015b). In VLS growth, nanowire diameters and morphology can be controlled by the dimension of the catalyst (Barth et al. 2011). Due to the direct relationship between the diameter of nanoparticle seeds and the diameter and morphology of semiconductor nanowires grown from them, precise control over the diameter and morphology of nanoparticle seeds, such as indium, is important (Wang et al. 2006; Barth et al. 2011; Chockla et al. 2011; Guillemin et al. 2012). In addition to the synthesis of III-V semiconductor nanowires, ligand-free indium nanoparticles have also been successfully used as heterogeneous catalysts in the allylation of various carbonyl compounds (Dorn et al. 2016). Moreover, individual indium nanoparticles have been used for fabricating single-electron transistors which may be utilised in future nanometre-scale electronic devices (Junno et al. 1999).

Due to the diverse application of indium nanoparticles, their synthesis with different size distributions and morphologies has been reported using various methods, such as the reduction of indium salts with $\mathrm{NaBH}_{4}$ (Chou 
et al. 2008; Hammarberg and Feldmann 2009; Kind and Feldmann 2011; George et al. 2015), $\mathrm{LiBH}_{4}$ (Lim et al. 2010), sodium metal (Khanna et al. 2005) or alkalides and electrides (Tsai and Dye 1991). These reduction approaches at high temperatures result in a highly sizemonodisperse spherical nanoparticles. In a two-step synthesis approach, the indium nanoparticles, initially synthesised by $\mathrm{NaBH}_{4}$ reduction of $\mathrm{InCl}_{3} \cdot 4 \mathrm{H}_{2} \mathrm{O}$ in diethylene glycol at $100{ }^{\circ} \mathrm{C}$, were transferred to a nonpolar medium via oleylamine (particle diameters between 10 and $12 \mathrm{~nm}$ ) (Hammarberg and Feldmann 2009). The synthesis of size-tunable (mean diameters between 8 and $105 \mathrm{~nm}$ ), air stable, citrate-capped indium nanoparticles, via $\mathrm{NaBH}_{4}$ reduction of $\mathrm{InCl}_{3} .4 \mathrm{H}_{2} \mathrm{O}$ in diethylene glycol at $100{ }^{\circ} \mathrm{C}$ is another example of high-temperature synthesis (Kind and Feldmann 2011). Furthermore, sub-10 nm spherical indium nanoparticles with uniform size and shape, capped with oleylamine, trioctylphosphine (TOP) or trioctylphosphine oxide (TOPO) have been synthesised by $\mathrm{LiBH}_{4}$ reduction of $\mathrm{InCl}_{3}$ under reflux conditions in isobutylamine (Lim et al. 2010). In all reported examples, uniform sizemonodisperse nanoparticles were obtained by the reduction of an indium salt at high temperatures (Hammarberg and Feldmann 2009; Lim et al. 2010; Kind and Feldmann 2011). However, the synthesis of nanoparticles at elevated temperatures also increases the chance of nanoparticle oxidation (Vot et al. 2014; Dong et al. 2015). Another point associated with hightemperature synthesis is increased production cost at scale up (Masala and Seshadri 2004; Zhang et al. 2015a). In addition to the problems accompanying the synthesis of indium nanoparticles at high temperatures, due to the poor solubility of the indium salts in nonpolar solvents, there is no report describing single-step synthesis of indium nanoparticles in non-polar solvents (Chou et al. 2008; Hammarberg and Feldmann 2009; Lim et al. 2010; Kind and Feldmann 2011; George et al. 2015). The only example of colloidal solutions of indium nanoparticles in non-polar solvents such as pentane or dodecane has been reported involving a two-step synthesis method, via the phase transfer of initially synthesised indium nanoparticles in a polar solvent with particle diameters between 10 and $12 \mathrm{~nm}$. The report also lacks data on the degradation stability of the nanoparticles in non-polar solvents which is important for further applications (Hammarberg and Feldmann 2009). However, the initial formation of nanoparticles in a refluxing polar solvent followed by a time-consuming phase transfer process renders the two-step approach inefficient.

Herein, we report the direct, single-step formation of highly stable size-monodispersed indium nanoparticles with a mean particle size of $6.4 \pm 0.4 \mathrm{~nm}$ in a non-polar medium through the reduction of anhydrous $\mathrm{InCl}_{3}$ with $\mathrm{LiBH}_{4}$ at room temperature using an innovative simultaneous phase transfer and ripening method. According to dynamic light scattering (DLS) analysis, the solution of indium nanoparticles does not show any aggregation even after 2 months. The described method should also be applicable to the direct, room temperature synthesis of other metallic nanoparticles in non-polar media without the requirement for a separate phase transfer step.

\section{Experimental}

Synthesis

All chemicals were used as received, except solvents which were either degassed under nitrogen for $1 \mathrm{~h}$ or put under vacuum. Synthesis of the indium nanoparticles was performed under dynamic nitrogen purging conditions. Indium nanoparticles were synthesised by the reduction of anhydrous $\mathrm{InCl}_{3}(\mathrm{ABCR}$, $98 \%$ ) with a $2 \mathrm{M}$ solution of $\mathrm{LiBH}_{4}$ in tetrahydrofuran (THF) (Sigma-Aldrich) in the presence of tri$n$-butylphosphine (TBP) (Aldrich, 93.5\%) as a capping ligand in $N, N$-dimethylformamide (DMF) (MERCK, 99\%) as solvent at room temperature. Four hundred fifty microlitres $(1.80 \mathrm{mmol})$ of TBP was added to $4.5 \mathrm{ml}$ of a $40 \mathrm{mM}$ solution of $\mathrm{InCl}_{3}$ $(0.18 \mathrm{mmol})$ in DMF (in a Schlenk flask under dynamic nitrogen purging) and the solution mixture stirred for $15 \mathrm{~min}$. Afterwards, $220 \mu \mathrm{l}$ of a $2 \mathrm{M}$ solution of $\mathrm{LiBH}_{4}(0.44 \mathrm{mmol})$ in THF was added dropwise over $90 \mathrm{~s}$ to the solution under vigorous stirring. Stirring was continued for $10 \mathrm{~min}$ after which $1 \mathrm{ml}$ of the colourless solution was taken by syringe and injected very fast into each of the two non-polar systems and the whole mixture was stirred vigorously for $60 \mathrm{~min}$. Non-polar system I contained $1.5 \mathrm{ml} n$-dodecane (Sigma-Aldrich, 99\%) and $0.5 \mathrm{ml}$ oleylamine (Aldrich, technical grade, $70 \%$ ) while non-polar system II contained $1.5 \mathrm{ml} n$-dodecane (Sigma-Aldrich, 99\%), 0.5-ml oleylamine (Aldrich, technical grade, $70 \%)$ and $56-\mathrm{mg}(0.33 \mathrm{mmol}) \mathrm{di}-$ phenylamine (DPA) (Sigma-Aldrich, 99\%). 
Analytical tools

Small angle $X$-ray scattering ( $S A X S$ ) measurement was performed with a high-flux SAXSess camera (Anton Paar, Graz, Austria) connected to a Debyeflex 3003 Xray generator (GE-Electric, Germany), operating at $40 \mathrm{kV}$ and $50 \mathrm{~mA}$ with a sealed-tube $\mathrm{Cu}$ anode. The Goebel-mirror focused and Kratky-slit collimated X-ray beam was line shaped (17 mm horizontal dimension at the sample) and scattered radiation from non-polar systems I and II, measured in the transmission mode was recorded by a one-dimensional MYTHEN-1k microstrip solid-state detector (Dectris Switzerland), within a q-range (with $\mathrm{q}$ being the magnitude of the scattering vector) of 0.1 to $5 \mathrm{~nm}^{-1}$. Using $\mathrm{Cu} \mathrm{K} \alpha$ radiation of wavelength $0.154 \mathrm{~nm}$ and a sample-to-detector distance of $309 \mathrm{~mm}$, this corresponds to a total $2 \theta$ region of 0.14 to $7^{\circ}$, applying the conversion $q$ $\left[\mathrm{nm}^{-1}\right]=4 \pi(\sin \theta) / \lambda$ with $2 \theta$ being the scattering angle with respect to the incident beam and $\lambda$ the wavelength of the X-rays.

Samples were filled into a $1 \mathrm{~mm}$ (diameter) reusable quartz capillary with vacuum-tight sealing screw-caps at both ends. All measurements of the samples and blank were done with the capillary in vacuum and at $20^{\circ}$, with an exposure time of $10 \mathrm{~min}$ each. The scattering of the blank ( $n$-dodecane) was subtracted from the scattering of the non-polar systems I and II (dispersed in $n$ dodecane, concentration $=0.2 \mathrm{mM}$ ) after normalising both spectra to the same transmission. Backgroundcorrected scattering data of the samples were analysed by applying the indirect Fourier transformation method, which evaluates the particle distance distribution function $\mathrm{p}(\mathrm{r})$ in real space by using the program GIFT (Bergmann et al. 2000).

$D L S$ was performed on a Zetasizer Nano ZS from Malvern Instrument equipped with a He-Ne laser. The scattered light was recorded at a backscattering angle of $173^{\circ}$. Control of the instrument parameters such as cuvette position, temperature, measurement time and repetitions as well as data acquisition and analysis was achieved using Zetasizer Software V.6.12. A fluorescence quartz cuvette of $10 \times 10 \times 48 \mathrm{~mm}$ size (Hellma Analytics) was used for measurement of colloidal solution of nanoparticles.

Transmission electron microscopy (TEM) analysis was conducted on a JEOL 2100 operated at $200 \mathrm{kV}$ in bright-field condition for imaging. Two drops of the colloidal solution of indium nanoparticles were placed on the TEM grid under nitrogen purging and afterwards the grid was put under vacuum to remove any volatiles. The grid was kept under nitrogen inside a sealed vial and brought out of the vial just before analysis.

Energy dispersive spectroscopy (EDS) was carried out on FEI Helios NanoLab 600i operating at $20 \mathrm{kV}$ and $0.69 \mathrm{nA}$ equipped with Oxford X-Max 80 detector. The same TEM grid was used for EDS analysis.

Fourier-transform infrared spectroscopy (FT-IR) was performed on a Bruker Alpha FT-IR spectrometer equipped with Platinum ATR single reflection diamond. The spectra were acquired in a range between 3500 and $400 \mathrm{~cm}^{-1}$. To obtain the spectra of the nanoparticlecapped ligand, $2 \mathrm{ml}$ of the colloidal solution of indium nanoparticles was mixed with $14 \mathrm{ml}$ of DMF and $6 \mathrm{ml}$ of THF and the mixture was stirred for 2 min followed by centrifugation at $2000 \mathrm{rpm}$ for $5 \mathrm{~min}$. Afterwards, the solution was removed and the precipitate was mixed with $10 \mathrm{ml}$ of DMF and redispersed with ultrasonication. The solution mixture was then centrifuged for $6 \mathrm{~min}$ at $2000 \mathrm{rpm}$ and afterwards the solution was removed and finally, the precipitate was put under vacuum for $10 \mathrm{~min}$ to remove any volatiles. The precipitate was directly subjected to FT-IR spectroscopy. The FT-IR spectra of the pure compounds were obtained by directly putting them onto the diamond cell.

Nuclear magnetic resonance (NMR) spectroscopy was performed on a Varian INOVA $300\left({ }^{31} \mathrm{P}\right.$ 121.4 MHz) spectrometer. A capillary filled with $\mathrm{D}_{2} \mathrm{O}$ was used for deuterium lock.

\section{Results and discussion}

Figure 1a illustrates schematically the procedure for the formation of indium nanoparticles according to the present study. Reduced $\mathrm{InCl}_{3}$ (using $\mathrm{LiBH}_{4}$ in $\mathrm{DMF}$ in the presence of TBP at ambient temperature) was mixed with oleylamine/ $n$-dodecane under vigorous stirring, the colourless mixture turning to a dark brown/black solution. The mixture was then kept immobile to permit separation of the polar (DMF) from non-polar (oleylamine/ $n$-dodecane) solvents containing the indium nanoparticles. TBP was distributed between both solvent phases due to its miscibility with both DMF and $n$-dodecane.

As the solubility of metal ion precursors in a nonpolar medium is limited (Yang et al. 2011), $\mathrm{InCl}_{3}$ was dissolved in DMF as a polar solvent. After reduction of 
Addition of TBP stabilised $\ln (0)$ to oleylamine solution
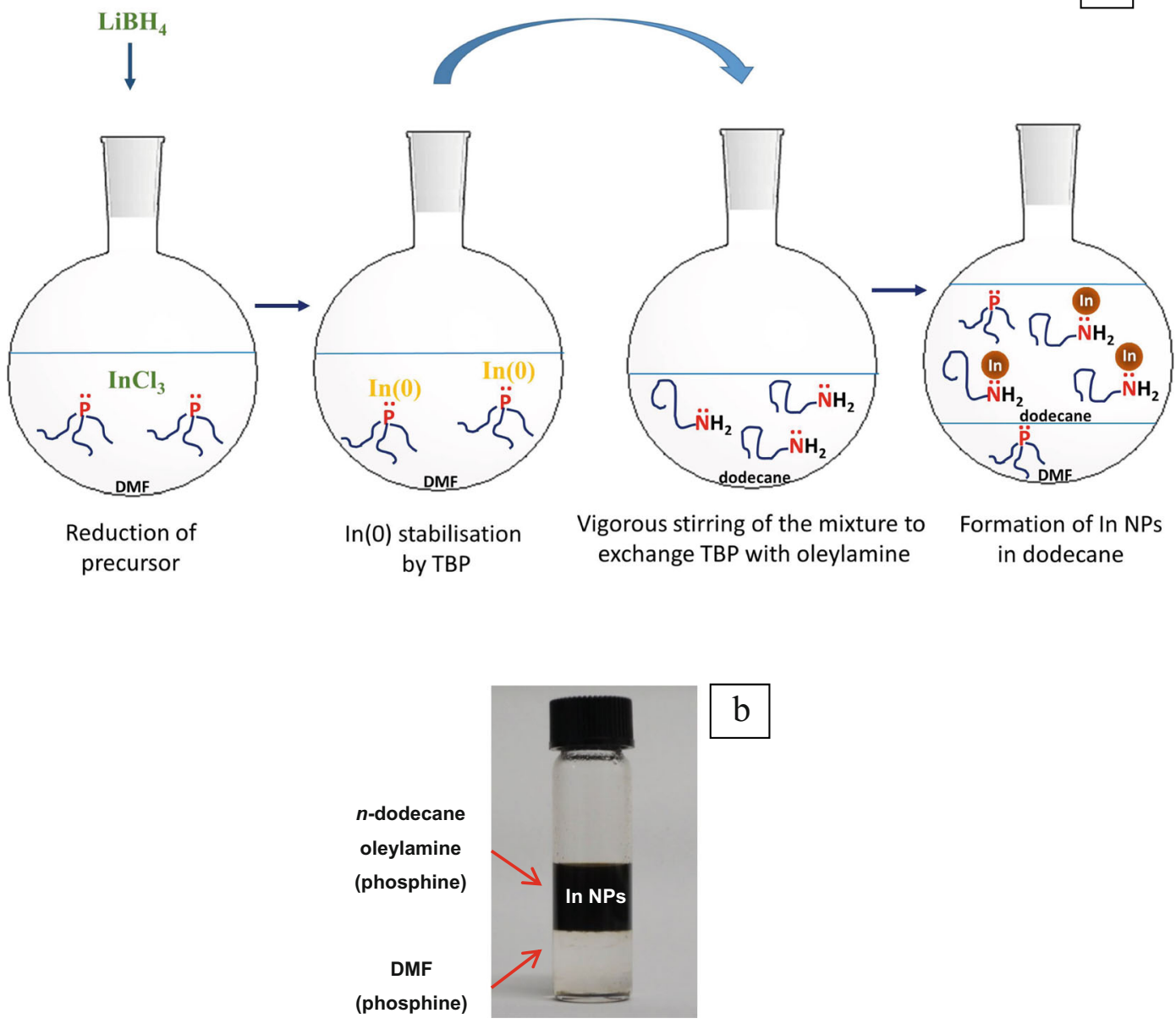

Fig. 1 a Schematic representation of the $\operatorname{In}(0)$ formation and stabilisation by TBP. TBP exchange by oleylamine under vigorous stirring transfers $\operatorname{In}(0)$ species to the non-polar solvent and finally the accumulation of $\operatorname{In}(0)$ in the non-polar solvent and formation of indium nanoparticles. b Indium nanoparticles after phase transfer. The bottom transparent layer is DMF and the top layer is a mixture of $n$-dodecane, oleylamine and indium nanoparticles
$\mathrm{InCl}_{3}$ with $\mathrm{LiBH}_{4}$ in DMF at room temperature, a stabiliser such as TBP is necessary to support the newly formed $\operatorname{In}(0)$ species in DMF and inhibit fast nucleation. The nucleation inhibiting behaviour of TBP can be observed from the colour change in the DMF solution. Colloidal solutions of sub-20 nm indium nanoparticles are typically dark brown in colour (Hammarberg and Feldmann 2009; Kind and Feldmann 2011). In contrast, the DMF solution containing TBP-stabilised In(0) species remains colourless for $1 \mathrm{~h}$ with a gradual colour change to pale yellow, which is a sign of $\operatorname{In}(0)$ nucleation. The solution turns a dark brown colour after stirring for $24 \mathrm{~h}$. TBP plays a key factor in the stabilisation of $\operatorname{In}(0)$ species as discrete entities in
DMF, acting as fast nucleation inhibitor. The absence of TBP results in the rapid nucleation of $\operatorname{In}(0)$ species and the formation of grey metallic indium particles. The slow nucleation of TBP-stabilised $\operatorname{In}(0)$ species and the very slow formation of indium nanoparticles in DMF, up to several hours, makes the transfer of discrete $\operatorname{In}(0)$ from DMF to a non-polar medium using a phase transfer reagent feasible. In this regard, before the nucleation of $\operatorname{In}(0)$ and the formation of indium nanoparticles in DMF, oleylamine transfers discrete $\operatorname{In}(0)$ species to a non-polar medium such as $n$-dodecane. In this case, vigorous stirring of the DMF solution containing $\operatorname{In}(0)$ mixed with oleylamine and $n$-dodecane promotes the transfer of discrete $\operatorname{In}(0)$ species from DMF to $n$-dodecane. In 
fact, instead of the previously reported phase transfer of nanoparticles from polar to non-polar medium (Hammarberg and Feldmann 2009), here, discrete TBP-stabilised $\operatorname{In}(0)$ species undergo the phase transfer reaction in the presence of oleylamine. Transfer of discrete $\operatorname{In}(0)$ to $n$-dodecane increases the concentration of $\operatorname{In}(0)$ species in $n$-dodecane which later promotes the nucleation and formation of indium nanoparticles in $n$ dodecane. In this regard, while the DMF solution of TBP-stabilised $\operatorname{In}(0)$ remains colourless for $1 \mathrm{~h}$ and turns to dark brown only after $24 \mathrm{~h}$, the addition of the solution of DMF containing TBP-stabilised $\operatorname{In}(0)$ to a mixture of oleylamine/ $n$-dodecane under vigorous stirring is accompanied by a rapid colour change from colourless to dark brown/black in less than $15 \mathrm{~min}$. This rapid colour change in the presence of oleylamine confirms that the discrete TBP-stabilised $\operatorname{In}(0)$ species, after phase transfer to $n$-dodecane, undergo fast nucleation resulting in the rapid formation of indium nanoparticles in the mixture of oleylamine/ $n$-dodecane.

Separation of the polar and non-polar media was achieved by immobilising the mixture for a minimum time of $1 \mathrm{~h}$; the non-polar medium forms the top layer, which due to the presence of indium nanoparticles is dark brown/black. During this process, the colourless DMF forms the bottom layer (Fig. 1b). Figure 2a shows DLS intensity distributions of the colloidal solutions of the indium nanoparticles in non-polar system I, i.e. only containing $n$-dodecane and oleylamine. The blue line represents the in situ prepared sample and the red line corresponds to the same sample after 7 days. DLS analysis of the in situ prepared sample showed that the indium nanoparticles had a mean diameter of $9 \mathrm{~nm}$. However, the colloidal solution was not stable over time and aggregation of nanoparticles occurred over a period of 7 days, as seen from the red curve which shifts towards a larger diameter regime. Conversely, Fig. $2 b$ shows the DLS intensity distribution of a colloidal solution of indium nanoparticles prepared using non-polar system II, i.e. with the additional presence of DPA along with $n$-dodecane and oleylamine. Again, the blue line represents the in situ prepared sample whereas the red line was obtained from the same sample aged after 70 days. The DLS traces of the in situ prepared samples were very similar for both non-polar systems I and II, with a mean diameter of $9 \mathrm{~nm}$ and no sign of agglomeration. However, despite the similarity of both in situ samples, over a large time interval, the nanoparticles synthesised through non-polar system I degraded and
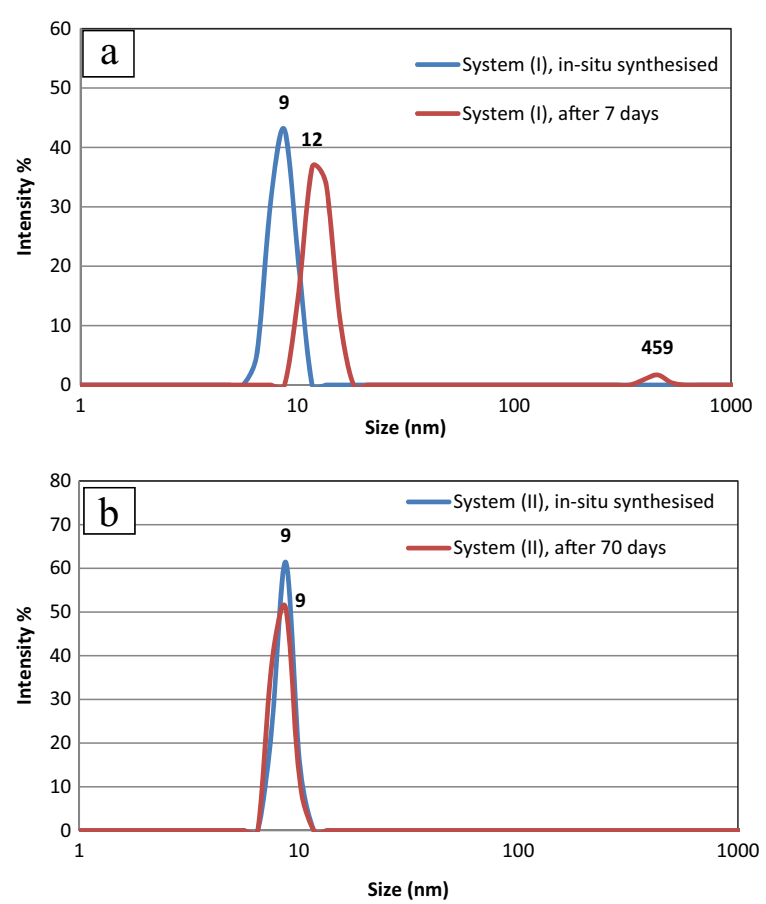

Fig. 2 DLS intensity distributions of colloidal solution of indium nanoparticles. a Non-polar system I. Blue line: in situ synthesised sample. Red line: the same sample after 7 days. Although nanoparticles are size-monodispersed without any sign of agglomeration for a fresh sample, agglomeration is clearly visible in the aged sample. b Non-polar system II. Blue line: in situ synthesised sample. Red line: the same sample after 70 days. Nanoparticles are size-monodispersed without any sign of agglomeration in both the fresh and the aged samples

showed signs of aggregation, whereas the nanoparticles obtained through non-polar system II remained stable even after 70 days (Fig. 2b). The only difference between non-polar systems I and II was the presence of DPA, which significantly improves the stability of the nanoparticles against aggregation.

The small angle X-ray scattering (SAXS) distance distribution functions (related to the second moment of the histogram of distances within the particles) of in situ prepared colloidal solutions of indium nanoparticles obtained using systems I and II are depicted in Fig. 3. The blue line corresponds to indium nanoparticles with oleylamine (system I) whereas the red line represents indium nanoparticles with oleylamine/DPA (system II). The additional green line exemplifies a theoretically calculated distance distribution function of sizemonodisperse spheres with symmetrical bell-shaped histogram and a mean diameter of $6.5 \mathrm{~nm}$. Indium nanoparticles synthesised via both systems displayed a mean particle diameter of $6.5 \mathrm{~nm}$ (radius between 3.0 


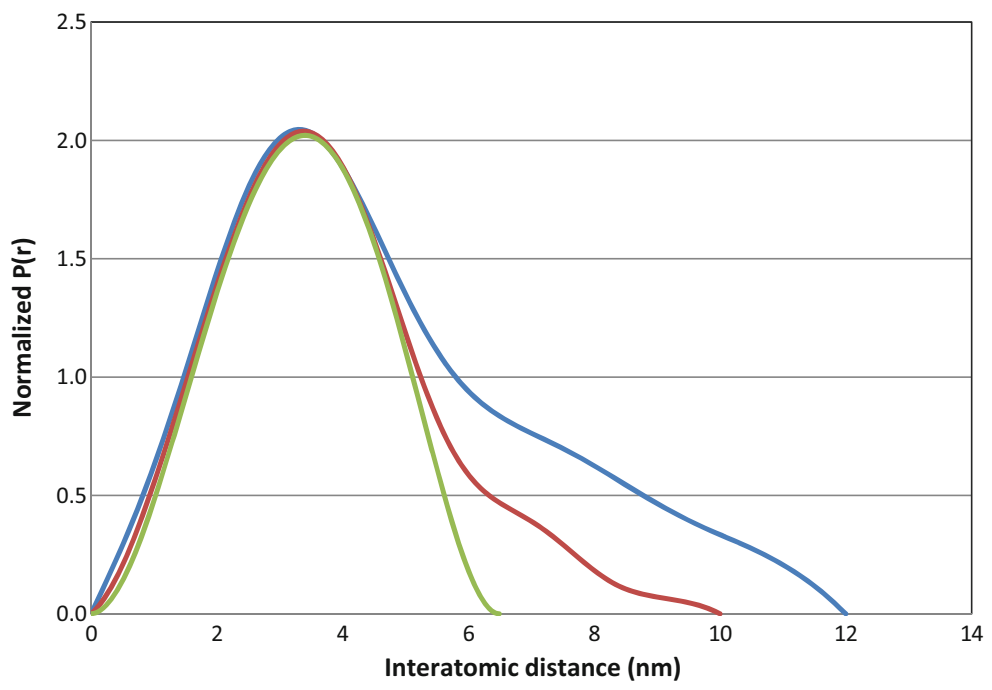

Fig. 3 Distance distribution function $p(r)$ of an in situ prepared colloidal solution of indium nanoparticles. Blue line: indium nanoparticles in the presence of oleylamine (system I). Red line: indium nanoparticles in the presence of oleylamine/DPA (system II). Green line: theoretically calculated distance distribution function

and $3.5 \mathrm{~nm}$ ) but also contained contributions of larger particle sizes (10 and $12 \mathrm{~nm}$, respectively). A comparison of the analytical data obtained from the SAXS and DLS techniques showed a difference of approximately $2 \mathrm{~nm}$ in the mean particle diameter. This difference in the mean particle diameter from both methods is due to the difference in the measurement techniques; SAXS data results from the electron density contrast between the sample and solvent (Schnablegger and Singh 2013) whereas DLS data are derived from the hydrodynamic diameter of the particles, which depends on the surface morphology of the particles (Lim et al. 2013) and any interactions between the particles and the medium (Kätzel 2007). Higher mean nanoparticle diameters from DLS measurements compared to SAXS analysis has previously been reported (Chen et al. 2012; Pabisch et al. 2012). However, DLS as a cheaper and quicker analysis technique is preferred over SAXS for the analysis of nanoparticles. According to the SAXS measurement shown in Fig. 3, the colloidal solution of indium nanoparticles synthesised via system I, with only oleylamine, exhibited a size distribution of particle diameters up to $12 \mathrm{~nm}$, whereas the colloidal solution of indium nanoparticles synthesised via system II with oleylamine/DPA was up to $10 \mathrm{~nm}$. The nanoparticle size distribution in the system I was broader than for nanoparticles synthesised with oleylamine/DPA (system II), displaying a narrower size distribution similar to a of a size-monodispersed sample with symmetrical bell-shaped histogram. The system II sample with oleylamine/DPA has a narrower distance distribution and contains sub-10 nm particles, compared to sub-12 $\mathrm{nm}$ particles for system I

monodispersed symmetrical bell-shaped histogram. This data suggests that besides improving the longterm stability of the nanoparticles, the addition of DPA also has a positive effect in obtaining a homogeneously distributed indium nanoparticles.

Figure $4 \mathrm{a}$ shows a bright-field TEM image of the indium nanoparticles using the system II approach with the nanoparticles' size histogram displaying a mean diameter of $6.4 \mathrm{~nm}$ and standard deviation of $0.4 \mathrm{~nm}$ (total number of nanoparticles analysed $=220$ ), confirming the sizemonodispersity of the sample. Large distances between individual nanoparticles indicated a welldispersed and non-agglomerated colloidal solution. According to the TEM image, the mean particle size was $6.4 \pm 0.4 \mathrm{~nm}$, which was in accordance with the SAXS data. The higher diameter value obtained from the DLS data compared to SAXS and TEM analysis can be attributed to the effect of the hydrodynamic shell around the particles in solution (Pabisch et al. 2012).

The formation of small nanoparticles with a narrow diameter distribution at room temperature deserves special attention. It is known that the synthesis of nanoparticles at high temperatures increases the rate of nucleation and thereby decrease the particle size (Kwon and Hyeon 2011). The synthesis of indium nanoparticles via the reduction of indium salts has been previously 
Fig. 4 a Bright-field TEM image of indium nanoparticles synthesised in the presence of oleylamine/DPA (system II), with a mean diameter of $6.4 \mathrm{~nm}$ (Total number of nanoparticles $=220$ ). Inset (left-down) shows nanoparticles in higher magnification, inset (right-up) shows histogram of the nanoparticles' diameter with the standard deviation of $0.4 \mathrm{~nm}$ and inset (left-up) shows SAED pattern of amorphous nanoparticles. b EDS analysis of indium nanoparticles. Presence of $\mathrm{Ni}$ and $\mathrm{Al}$ and carbon relates to the TEM grid
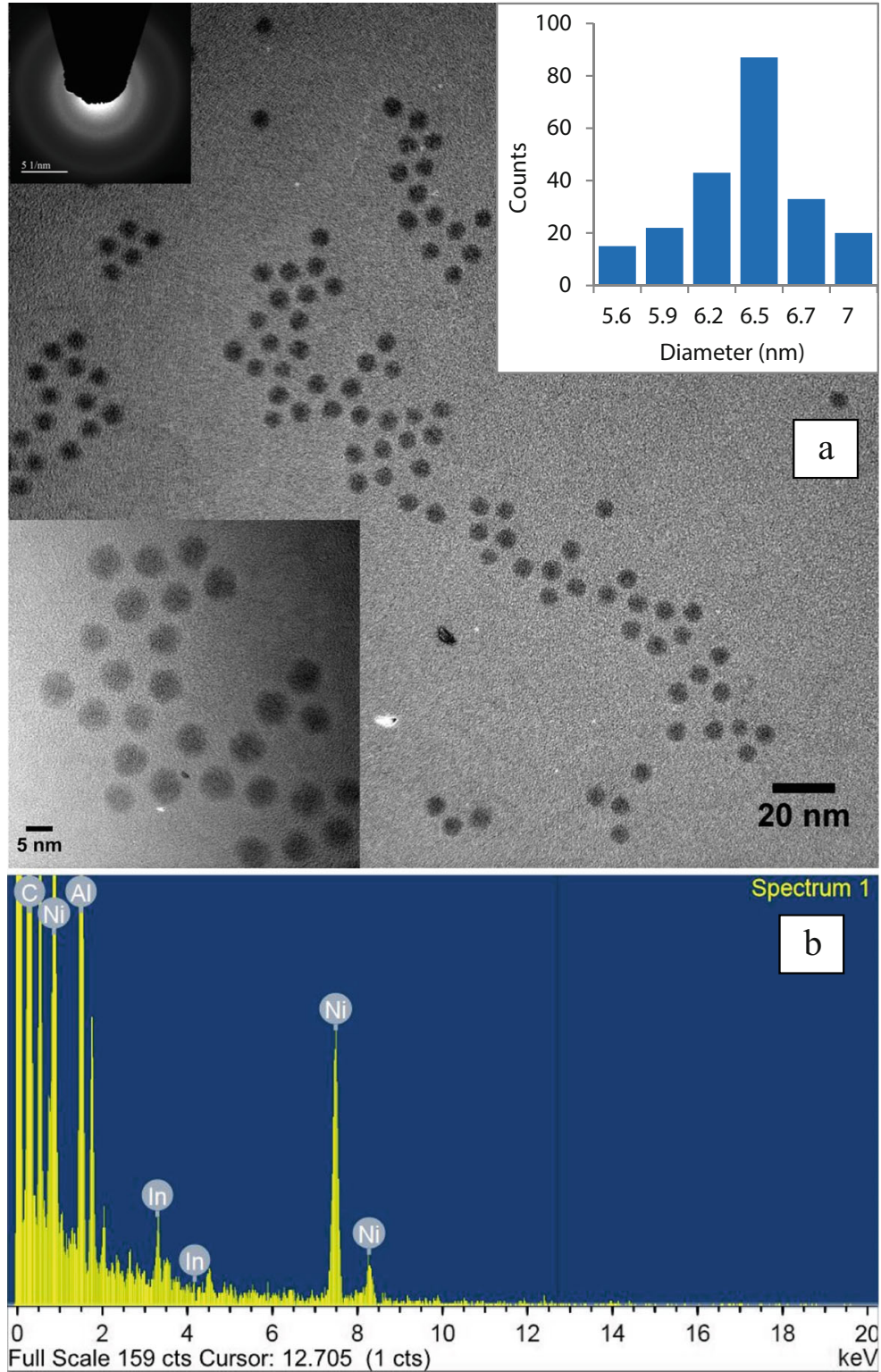

reported at $100{ }^{\circ} \mathrm{C}$ resulting in the formation of nanoparticles with diameters between 10 and $12 \mathrm{~nm}$ (Hammarberg and Feldmann 2009). In another approach, indium nanoparticles were synthesised in reflux with mean diameters of $7.1 \pm 0.5 \mathrm{~nm}, 7.8 \pm 0.3 \mathrm{~nm}$ and $8.1 \pm 1.1$ by varying the capping ligand (Lim et al. 2010). In contrast, the synthetic procedure reported here allows for the formation of small nanoparticles with a narrow diameter distribution at room temperature, i.e. $6.4 \pm 0.4 \mathrm{~nm}$. The small mean particle diameter obtained in our approach, compared to nanoparticles synthesised by standard high-temperature methods, can be attributed to the saturation of DMF with TBPstabilised $\operatorname{In}(0)$ species, where nucleation is inhibited by the stabilising effect of TBP. As soon as this solution is mixed with oleylamine/ $n$-dodecane, an instantaneous colour change to yellow takes place which can be attributed to the formation of an infinite number of nuclei in a short moment, known as burst of nucleation (Kwon and Hyeon 2011). Burst of nucleation is related to the availability of a high concentration of $\operatorname{In}(0)$ species (or solute saturation) which instantaneously increases the chance of infinite nucleation and hence reduction of mean particle size. Another advantage of burst 
nucleation, according to classical LaMer nucleation and growth theory, is the formation of size-monodisperse colloidal solution of nanoparticles (LaMer and Dinegar 1950; LaMer 1952). Nucleation and growth of particles according to the LaMer mechanism is divided into three separate steps: (i) the formation of a high concentration of free atoms in solution; (ii) instantaneous nucleation of the free atoms or a nucleation burst, after which point no further nucleation is favourable due to the reduced concentration of the free atoms and (iii) growth of the initially formed nuclei. Due to the consumption of most of the free atoms during the second step, the concentration of the remaining free atoms is insufficient for the initiation of any new nucleation events and thus according to the third step, the remaining free atoms inside the solution will be consumed for the growth of the initially formed nuclei (LaMer and Dinegar 1950; LaMer 1952). In the synthesis of our nanoparticles, the nucleation burst drastically reduces the $\operatorname{In}(0)$ species in solution which hinders further nucleation. As a result the remaining $\operatorname{In}(0)$ will be consumed only for the growth of the initially formed nuclei. In general, the availability of the infinite TBP-stabilised $\operatorname{In}(0)$ species in DMF is the key factor for the nucleation burst when the DMF solution is mixed with oleylamine/ $n$-dodecane and hence the formation of small nanoparticles with a narrow diameter distribution.

The relationship between the polarity of the solvent, or the capping ligand, and the morphology of nanocrystals and nanoparticles has been extensively studied (Sau and Murphy 2004; Khoza et al. 2012; Eastoe and Tabor 2014).
The interaction between a solvent or capping ligand and the crystal interface determines the crystal growth rate in a preferential direction. High polarity solvents or capping ligands result in increased interaction with a particular interface inducing uniaxial growth (Sau and Murphy 2004; Xu et al. 2009; Xiao and Qi 2011). However, low polarity solvents or capping ligands promote isotropic and spherical growth of the crystals (Xu et al. 2009). As shown in Fig. 4a, the spherical shape of the indium nanoparticles produced in this study is due to the growth in a non-polar solvent, with minimum interaction between the solvent and the interfaces of the nanoparticles. Moreover, the growth of spherical nanoparticles highlights the isotropic interaction between the interface of the indium nanoparticles and oleylamine as the capping ligand.

Figure 4a (left-top inset) shows an SAED pattern of the nanoparticles with amorphous structure. The presence of amorphous nanoparticles is expected with room temperature synthesis and has been reported elsewhere (Soulantica et al. 2001). Figure 4b displays EDS analysis of indium nanoparticles showing the presence of characteristic indium peaks. The presence of $\mathrm{Al}, \mathrm{Ni}$ and carbon in the EDS spectrum relates to the TEM grid.

As evolution and ripening of indium nanoparticles occurs in a solution mixture containing TBP, oleylamine and DPA and as the ${ }^{31} \mathrm{P}$ NMR spectroscopic analysis showed that TBP presented in both DMF and oleylamine $/ n$-dodecane mixture after formation of the nanoparticles, it is important to determine what type of capping ligand or what mixture of them is attached to the indium nanoparticles. Figure 5 shows FT-IR spectra
Fig. 5 FT-IR spectra of pure DPA (yellow line), pure oleylamine (red line), indium nanoparticles synthesised from $n$ dodecane/oleylamine-DPA (system II) (violet line), indium nanoparticles from $n$-dodecane/ oleylamine (system I) (blue line) and pure TBP (green line)

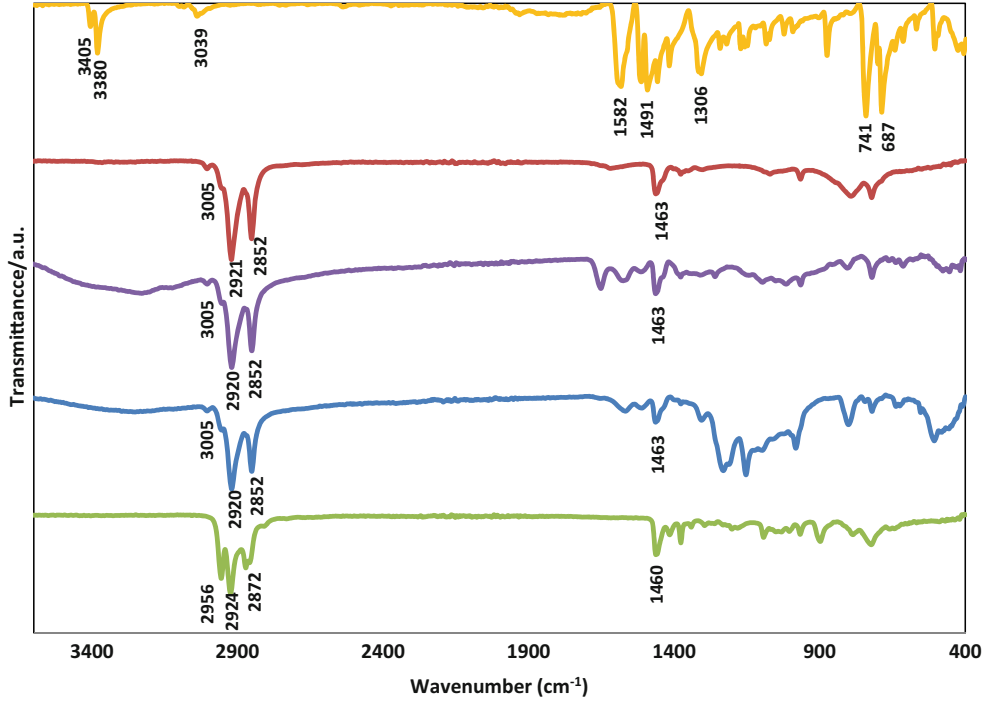


of indium nanoparticles synthesised in a mixture of oleylamine/ $n$-dodecane/DPA (system II, violet line) and oleylamine/ $n$-dodecane mixture (system I, blue line) and compared with pure oleylamine (red line) and pure TBP (green line). Characteristic IR vibrations of pure oleylamine at 3005, 2921, 2852, $1463 \mathrm{~cm}^{-1}$ were similar to both samples which were prepared with oleylamine and an oleylamine/DPA mixture. Although initially TBP stabilises $\operatorname{In}(0)$ in DMF, after the phase transfer reaction, TBP was not visible in the FT-IR spectrum, instead, oleylamine was present around indium nanoparticles.

For the non-polar system II, DPA is less basic than oleylamine due to the presence of electron withdrawing aromatic groups (Dewick 2013). Therefore, oleylamine is a better donor to indium nanoparticles than DPA. This can be confirmed by the absence of DPA in the FT-IR spectra of the indium nanoparticles (Fig. 5). Although DPA does not act as capping ligand, its presence in the solution mixture drastically improves the sizemonodispersity and the long-term stability of the colloidal solution according to DLS results in Fig. $2 b$ and TEM results in Fig. 4a. A credible role for stabilisation of nanoparticles with DPA can be its effect as a barrier between nanoparticles. To further study this effect, the amount of DPA was increased to 80 from $56 \mathrm{mg}$ in an oleylamine $/ n$-dodecane mixture $(0.5 \mathrm{ml} / 1.5 \mathrm{ml})$. The solubility of $80 \mathrm{mg}$ of DPA in solution was found to be the maximum solubility of DPA in the mentioned mixture. In this case, while the saturation of the solution mixture was achieved with DPA, precipitation of the indium nanoparticles out of the solution mixture was observed during the formation of indium nanoparticles. This precipitation of indium nanoparticles happened as the solution mixture was completely saturated with DPA thus providing no more space inside the solution for further accommodation of the bulky indium nanoparticles. This observation further suggests the role DPA plays as a barrier between nanoparticles.

\section{Conclusions}

The synthesis of indium nanoparticles directly in a nonpolar solvent and at room temperature was achieved using a new simultaneous phase transfer and ripening method, based on the phase transfer of single metallic atoms instead of bulky nanoparticles. Although the reduction of the indium precursor and the synthesis of nanoparticles occurred at room temperature, fine nanoparticles with the mean diameter of $6.4 \pm 0.4 \mathrm{~nm}$ were obtained. The key factor for obtaining small diameter, size-monodisperse nanoparticles is due to the formation of TBP-stabilised $\operatorname{In}(0)$ species which trigger the burst nucleation and formation of fine nanoparticles. The addition of DPA decreased the distance distribution function of the nanoparticles, according to SAXS analysis, and improves the durability of the colloidal solution of nanoparticles to more than 2 months. This technique could be used for the synthesis of a broad range of metallic nanoparticles.

Acknowledgements Open access funding provided by Austrian Science Fund (FWF). Support for this study was provided by the Austrian Fonds zur Förderung der wissenschaftlichen Forschung (FWF) via the projects P-22678 and P-26417 and Science Foundation Ireland (Grant: 14/IA/2513). The authors are grateful to Prof. Dr. Torsten Mayr and Dr. Elisabeth Scheucher (Institute of Analytical Chemistry and Food Chemistry, Graz University of Technology, Graz, Austria) for their support and the use of the Zetasizer Nano ZS instrument. Also, Dr. Amin Sadeghpour (Department of Chemistry, Karl-Franzens-University Graz, Graz, Austria) is gratefully acknowledged for his kind introduction to the SAXS and DLS method.

\section{Compliance with ethical standards}

Conflict of interest The authors declare that they have no conflict of interest.

Open Access This article is distributed under the terms of the Creative Commons Attribution 4.0 International License (http:// creativecommons.org/licenses/by/4.0/), which permits unrestricted use, distribution, and reproduction in any medium, provided you give appropriate credit to the original author(s) and the source, provide a link to the Creative Commons license, and indicate if changes were made.

\section{References}

Barth S, Koleśnik MM, Donegan K et al (2011) Diametercontrolled solid-phase seeding of germanium nanowires: structural characterization and electrical transport properties. Chem Mater 23:3335-3340. doi:10.1021/cm200646e

Bergmann A, Fritz G, Glatter O (2000) Solving the generalized indirect Fourier transformation (GIFT) by Boltzmann simplex simulated annealing (BSSA). J Appl Crystallogr 33: 1212-1216. doi:10.1107/S0021889800008372

Chen ZH, Kim C, Zeng X et al (2012) Characterizing size and porosity of hollow nanoparticles: SAXS, SANS, TEM, DLS, and adsorption isotherms compared. Langmuir 28:1535015361. doi:10.1021/la302236u 
Chockla AM, Harris JT, Korgel BA (2011) Colloidal synthesis of germanium nanorods. Chem Mater 23:1964-1970. doi:10.1021/cm2001607

Chou NH, Ke X, Schiffer P, Schaak RE (2008) Room-temperature chemical synthesis of shape-controlled indium nanoparticles. J Am Chem Soc 130:8140-8141. doi:10.1021/ja801949c

Dewick PM (2013) Essentials of organic chemistry: for students of pharmacy, medicinal chemistry and biological chemistry. Wiley, New York

Dong H, Chen Y-C, Feldmann C (2015) Polyol synthesis of nanoparticles: status and options regarding metals, oxides, chalcogenides, and non-metal elements. Green Chem 17: 4107-4132. doi:10.1039/C5GC00943J

Dorn V, Chopa A, Radivoy G (2016) Mild bottom-up synthesis of indium(0) nanoparticles: characterization and application in the allylation of carbonyl compounds. RSC Adv 6:2379823803. doi:10.1039/C5RA27928C

Eastoe J, Tabor RF (2014) Surfactants and nanoscience. In: Colloidal foundations of nanoscience. Elsevier, Amsterdam, pp. 135-157

George A, Choudhary HK, Satpati B, Mandal S (2015) Synthesis, characterization and optical properties of ligand-protected indium nanoparticles. Phys Chem Chem Phys 17:71097113. doi:10.1039/C4CP05743K

Guillemin S, Consonni V, Appert E et al (2012) Critical nucleation effects on the structural relationship between $\mathrm{ZnO}$ seed layer and nanowires. J Phys Chem C 116:25106-25111. doi:10.1021/jp308643w

Hammarberg E, Feldmann C (2009) In0 nanoparticle synthesis assisted by phase-transfer reaction. Chem Mater 21:771-774. doi:10.1021/cm803090e

Junno T, Magnusson MH, Carlsson S-B et al (1999) Singleelectron devices via controlled assembly of designed nanoparticles. Microelectron Eng 47:179-183. doi:10.1016/S0167-9317(99)00184-7

Kätzel U (2007) Dynamic light scattering for the characterization of polydisperse fractal systems by the example of. Unpublished $\mathrm{PhD}$ dissertation, Dresden University of Technology

Khanna PK, Jun K-W, Hong KB et al (2005) Colloidal synthesis of indium nanoparticles by sodium reduction method. Mater Lett 59:1032-1036. doi:10.1016/j.matlet.2004.11.050

Khoza PB, Moloto MJ, Sikhwivhilu LM (2012) The effect of solvents, acetone, water, and ethanol, on the morphological and optical properties of $\mathrm{ZnO}$ nanoparticles prepared by microwave. J Nanotechnol 2012:e195106. doi:10.1155/2012/195106

Kind C, Feldmann C (2011) One-pot synthesis of In0 nanoparticles with tuned particle size and high oxidation stability. Chem Mater 23:4982-4987. doi:10.1021/cm202256t

Kwon SG, Hyeon T (2011) Formation mechanisms of uniform nanocrystals via hot-injection and heat-up methods. Small 7: 2685-2702. doi:10.1002/smll.201002022

LaMer VK (1952) Nucleation in phase transitions. Ind Eng Chem 44:1270-1277. doi:10.1021/ie50510a027

LaMer VK, Dinegar RH (1950) Theory, production and mechanism of formation of monodispersed hydrosols. J Am Chem Soc 72:4847-4854. doi:10.1021/ja01167a001
Lim TH, Ingham B, Kamarudin KH et al (2010) Solution synthesis of monodisperse indium nanoparticles and highly faceted indium polyhedra. Cryst Growth Des 10:3854-3858. doi: $10.1021 / \operatorname{cg} 100857 y$

Lim J, Yeap SP, Che HX, Low SC (2013) Characterization of magnetic nanoparticle by dynamic light scattering. Nanoscale Res Lett 8:381. doi:10.1186/1556-276X-8-381

Masala O, Seshadri R (2004) Synthesis routes for large volumes of nanoparticles. Annu Rev Mater Res 34:41-81. doi:10.1146/annurev.matsci.34.052803.090949

Pabisch S, Feichtenschlager B, Kickelbick G, Peterlik H (2012) Effect of interparticle interactions on size determination of zirconia and silica based systems - a comparison of SAXS, DLS, BET, XRD and TEM. Chem Phys Lett 521:91-97. doi:10.1016/j.cplett.2011.11.049

Sau TK, Murphy CJ (2004) Room temperature, high-yield synthesis of multiple shapes of gold nanoparticles in aqueous solution. J Am Chem Soc 126:8648-8649. doi:10.1021/ja047846d

Schnablegger H, Singh Y (2013) The SAXS Guide. Anton Paar $\mathrm{GmbH}$

Soulantica K, Maisonnat A, Fromen M-C et al (2001) Synthesis and self-assembly of monodisperse indium nanoparticles prepared from the organometallic precursor. Angew Chem Int Ed Engl 40:448-451

Strupeit T, Klinke C, Kornowski A, Weller H (2009) Synthesis of InP nanoneedles and their use as Schottky devices. ACS Nano 3:668-672. doi:10.1021/nn800846d

Tsai KL, Dye JL (1991) Nanoscale metal particles by homogeneous reduction with alkalides or electrides. J Am Chem Soc 113:1650-1652. doi:10.1021/ja00005a031

Vot SL, Dambournet D, Groult H et al (2014) Synthesis of tin nanocrystals in room temperature ionic liquids. Dalton Trans 43:18025-18034. doi:10.1039/C4DT02289K

Wang F, Dong A, Sun J et al (2006) Solution-liquid-olid growth of semiconductor nanowires. Inorg Chem 45:7511-7521. doi:10.1021/ic060498r

Xiao J, Qi L (2011) Surfactant-assisted, shape-controlled synthesis of gold nanocrystals. Nanoscale 3:1383. doi:10.1039/c0nr00814a

$\mathrm{Xu} \mathrm{L}, \mathrm{Hu}$ Y-L, Pelligra $\mathrm{C}$ et al (2009) $\mathrm{ZnO}$ with different morphologies synthesized by solvothermal methods for enhanced photocatalytic activity. Chem Mater 21:2875-2885. doi:10.1021/cm900608d

Yang J, Lee JY, Ying JY (2011) Phase transfer and its applications in nanotechnology. Chem Soc Rev 40:1672-1696. doi:10.1039/B916790K

Zhang G, Li J, Zhang G, Zhao L (2015a) Room-temperature synthesis of Ni nanoparticles as the absorbent used for sewage treatment. Adv Mater Sci Eng 2015:e973648. doi: $10.1155 / 2015 / 973648$

Zhang G, Rainville C, Salmon A et al (2015b) Bridging the gap between the nanometer-scale bottom-up and micrometer-scale top-down approaches for site-defined InP/InAs nanowires. ACS Nano 9:10580-10589. doi:10.1021/acsnano.5b03682 